\title{
Reconstruction of Attosecond Pulse Trains Using an Adiabatic Phase Expansion
}

\author{
K. Varjú, ${ }^{1, *}$ Y. Mairesse, ${ }^{2}$ P. Agostini, ${ }^{2}$ P. Breger,${ }^{2}$ B. Carré,${ }^{2}$ L. J. Frasinski, ${ }^{3}$ E. Gustafsson, ${ }^{1}$ P. Johnsson, ${ }^{1}$ J. Mauritsson, ${ }^{1, \dagger}$ \\ H. Merdji, ${ }^{2}$ P. Monchicourt, ${ }^{2}$ A. L'Huillier, ${ }^{1}$ and P. Salières ${ }^{2}$ \\ ${ }^{1}$ Department of Physics, Lund University, P.O. Box 118, SE-221 00 Lund, Sweden \\ ${ }^{2}$ Service des Photons, Atomes et Molécules, CEA Saclay, 91191 Gif-sur-Yvette, France \\ ${ }^{3}$ J. J. Thomson Physical Laboratory, The University of Reading, Whiteknights, P.O. Box 220, Reading RG6 6AF, United Kingdom
}

(Received 28 June 2005; published 6 December 2005)

\begin{abstract}
We propose a new method to reconstruct the electric field of attosecond pulse trains. The phase of the high-order harmonic emission electric field is Taylor expanded around the maximum of the laser pulse envelope in the time domain and around the central harmonic in the frequency domain. Experimental measurements allow us to determine the coefficients of this expansion and to characterize the radiation with attosecond accuracy over a femtosecond time scale. The method gives access to pulse-to-pulse variations along the train, including the timing, the chirp, and the attosecond carrier envelope phase.
\end{abstract}

When an intense laser field interacts with a gas, highorder harmonics are emitted in subfemtosecond bursts of light $[1,2]$. The temporal characterization of this radiation is of great fundamental interest since it gives insight into the emission process. In addition, the ultrashort duration corresponding to a selected bandwidth makes this radiation a unique extreme ultraviolet (XUV) source of interest for a number of applications.

Individual harmonic pulses can be characterized on the femtosecond time scale by techniques such as FROG (frequency-resolved optical gating) [3-7] and SPIDER (spectral phase interferometry for direct electric field reconstruction) $[8,9]$. Attosecond pulses are characterized by recently developed techniques $[2,10]$ such as RABITT (reconstruction of attosecond beating by interference of two-photon transition). The latter shows the existence of a quadratic spectral phase, i.e., a frequency chirp [11,12] (hereafter called attochirp) [13], for the plateau harmonics.

The RABITT technique, which assumes monochromatic harmonic components, gives access only to the average pulse shape in the train. New measurement techniques, based on extensions of the FROG method, have been proposed and numerically verified [14-16]. All these methods imply a scan of an optical delay, and the reconstruction of a complete attosecond pulse train (APT) demands a temporal accuracy of a few tens of attoseconds over several tens of femtoseconds, which is difficult to achieve experimentally.

In this Letter, we propose a new technique that, by making physically reasonable assumptions, practically removes the high requirement on the experiment. The ultraprecise scan is replaced by a series of short scan RABITT measurements performed at different laser intensities. The reconstruction of the electric field uses a Taylor expansion of the harmonic phase with respect to frequency and time whose coefficients have simple physical interpretations. The method is illustrated by characterizing an APT generated in neon.
We consider a coherent sum of consecutive odd harmonics (from $q_{i}$ to $q_{f}$ ) generated when a strong laser field interacts with a gas. We assume for simplicity that only one quantum path, selected by either phase matching or an aperture in the far field [17-20], contributes to the generation of these harmonics. The electric field resulting from the superposition of these harmonics can be written as

$$
E(t)=\sum_{q=q_{i}}^{q=q_{f}} A_{q}(t) e^{-i \Psi_{q}(t)},
$$

where $\omega$ is the laser frequency and $A_{q}(t)$ and $\Psi_{q}(t)$ are the amplitude and phase of the $q$ th harmonic field. The phase $\Psi_{q}(t)=q \Phi_{0}(t)-\Phi_{q}(t)-\Phi_{q}^{\text {prop }}$ includes a contribution from the fundamental field $q \Phi_{0}(t)$, with $\Phi_{0}(t)=$ $\omega t+\phi_{0}+b_{0} t^{2} / 2$ where $\phi_{0}$ is the fundamental pulse carrier envelope phase (CEP) and $b_{0}$ a possible frequency chirp, as well as phase terms originating from the generation process $\Phi_{q}$ and dispersive propagation effects $\Phi_{q}^{\text {prop }}$. $|E(t)|^{2}$ typically consists of a train of pulses of a few hundred attoseconds in duration, separated by half the laser period, spanning over an interval of a few tens of femtoseconds. Interestingly, in Eq. (1) we treat the two time scales of the problem differently: the femtosecond structure is expressed in the time domain, through the (slow) time variation of $A_{q}(t)$ and $\Psi_{q}(t)-q \omega t$, while the attosecond structure is here characterized by the spectral variation of $A_{q}$ and $\Psi_{q}$.

Our reconstruction of the APT uses a Taylor expansion up to fourth order of the phase term both in frequency $\Omega$, covering the whole frequency range by discrete steps of $2 \omega$ and in time on the femtosecond time scale. The Taylor expansion is performed around $(\Omega, t)=\left(q_{0} \omega, 0\right)$, where $q_{0}$ is the central harmonic order and $t=0$ is the maximum of the laser pulse. The phase term originating from the generation process $\Phi_{q}(t)$ depends on time via the fundamental laser intensity envelope $I(t)$ [21]. Assuming that $I(t)$ is 
symmetric with respect to $t=0$, all odd derivatives around $t=0$ are equal to zero. The third and fourth order terms in $\left(\Omega-q_{0} \omega\right)$, as well as the fourth order terms in $t$ are found to be very small and can be neglected. We use the notation $\gamma=d^{2} I / d t^{2}$, at $t=0$. For a Gaussian pulse characterized by a peak intensity $I_{0}$ and a pulse duration $\tau, \gamma=$ $-8 \ln (2) I_{0} / \tau^{2}$. Writing the frequency derivatives as $\partial \Phi / \partial \Omega=1 / \omega \partial \Phi / \partial q$, we obtain

$$
\begin{aligned}
\Psi_{q}(t) \approx & q_{0} \omega t-\Phi_{q_{0}}\left(I_{0}\right)+q_{0} \phi_{0}+\left(q_{0} \frac{b_{0}}{2}-\frac{\gamma}{2} \frac{\partial \Phi}{\partial I}\right) t^{2} \\
& +\left(\omega t+\phi_{0}-\frac{\partial \Phi}{\partial q}\right)\left(q-q_{0}\right)-\left(\frac{1}{2} \frac{\partial^{2} \Phi}{\partial q^{2}}+\delta \frac{\omega^{2}}{2}\right)\left(q-q_{0}\right)^{2} \\
& +\left(\frac{b_{0}}{2}-\frac{\gamma}{2} \frac{\partial^{2} \Phi}{\partial q \partial I}\right)\left(q-q_{0}\right) t^{2}-\left(\frac{\gamma}{4} \frac{\partial^{3} \Phi}{\partial q^{2} \partial I}\right)\left(q-q_{0}\right)^{2} t^{2} .
\end{aligned}
$$

All the partial derivatives are taken at $q=q_{0}$ and $I=I_{0}$. The effect induced by propagation through a medium is taken into account by a constant group delay dispersion, $\delta$.

All the terms in Eq. (2) have a simple physical interpretation. The first line describes the phase of the carrier of the APT electric field relative to the envelope of the fundamental field. It does not depend explicitly on $q$ and will be factored out of the sum in Eq. (1). The second line in Eq. (2) describes the attosecond pulse intensity characteristics at the peak of the laser pulse. The first term expresses the delay of the attosecond burst with respect to the fundamental envelope and the second gives the variation of this delay as function of frequency, i.e., the attochirp. Finally, the last line in the equation describes how the delay and delay dispersion vary with the laser intensity, and hence with time, on a femtosecond time scale. Equation (2) clarifies the degrees of freedom available for tailoring the APT. The fundamental chirp $b_{0}$ affects the spacing of the bursts in the train [7], while the material group delay dispersion, $\delta$, affects the chirp of the attosecond pulses [17].

Our proposal for reconstruction of the APT is based on the experimental determination of the spectral amplitudes $A_{q}(I)$, the first derivative of the phase with respect to intensity, the first and second order derivatives of the phase with respect to $q$, and their variation with intensity. These measurements allow for a complete characterization of the APT except for a (constant) phase term. Our reconstruction method assumes that the XUV field is determined by the local (fs) laser intensity and phase and therefore applies to relatively long and low intensity generating laser pulses for which nonadiabatic effects are negligible. The term $\partial \Phi / \partial I$, which is not required for determination of the APT intensity profile, can be determined by measurements giving access to the chirp of the central harmonic of the generated spectrum $[7,8]$. The derivatives appearing in the last two lines of Eq. (2) can all be determined from RABITT measurements [11,18], performed at different generating intensities.

In RABITT, atoms are photoionized by a superposition of the APT and the optically delayed fundamental field. The photoelectron spectra display, between each onephoton harmonic line, two-photon sidebands induced by absorption of one harmonic and absorption or emission of a laser photon. When scanning the delay, the sidebands amplitudes oscillate with half the fundamental period and the phase of each oscillation allows us to retrieve the phase difference of the corresponding harmonics pair $\Phi_{q+2}-$ $\Phi_{q} \approx 2 \partial \Phi / \partial q$. The RABITT reconstruction is based on the assumption of an infinite driving pulse. Therefore measurements involving finite pulses intrinsically involve spatial and temporal averaging over a sufficient number of oscillations. To test the effect of temporal averaging, we have simulated a RABITT measurement and reconstruction, using a typical APT generated with a 45 fs pulse. We find that the determined derivatives compare well with the values obtained at a fixed intensity, slightly lower than the peak intensity.

To verify the consistency of the proposed reconstruction method, we present results from an experiment carried out at the LUCA facility in Saclay, using the arrangement described in Ref. [11]. High-order harmonics were generated with $45 \mathrm{fs}, 800 \mathrm{~nm}$ laser pulses. A series of RABITT measurements were performed in neon over a spectral range from the 19th to the 37th harmonics at several laser intensities ranging from 2 to $4 \times 10^{14} \mathrm{~W} / \mathrm{cm}^{2}$. We plot in Fig. 1(a) $\partial \Phi / \partial q$ values obtained by analyzing the positions of the oscillations of the sidebands as a function of harmonic order. Both $\partial \Phi / \partial q$ (group delay) and $\partial^{2} \Phi / \partial q^{2}$ (group delay dispersion) are positive and decrease as the laser intensity increases. In Fig. 1(b), we plot the reconstructed average pulses, obtained from the RABITT algorithm $[2,11]$, which essentially amounts to neglecting the time dependence in $A_{q}$ and $\Phi_{q}$. The pulse duration, as well as the delay relative to the fundamental field, decreases significantly with increasing intensity. As a consequence, the different pulses of an APT present similar distortions, since they are generated at different intensities: the pulses are shorter at the maximum of the train, and the spacing between them increases along the train. In essence, the pulses reconstructed at different peak intensities [cf. Fig. 1(b)] mirror the temporal variation of the pulses in the train generated at the highest intensity.

Strong field approximation (SFA) [13,21] calculations of the single-atom response have been carried out by solving saddle point equations for the experimental conditions. 

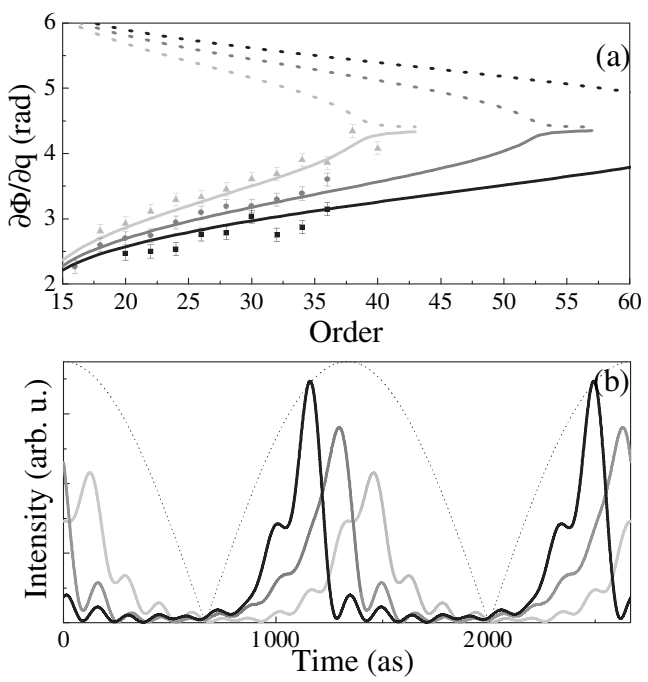

FIG. 1. (a) Variation of the phase derivative $\partial \Phi / \partial q$ with harmonic order $q$ at laser intensities of 2 (light gray), 3 (dark gray), and $4 \times 10^{14} \mathrm{~W} / \mathrm{cm}^{2}$ (black) in neon. The markers show experimental data obtained from RABITT measurements. The solid and dashed lines are the SFA predictions for the short and long trajectories. (b) Corresponding reconstructed average attosecond pulses for harmonics 19 to 37. The pulses have been normalized to contain the same energy. The dashed line is the absolute value of the fundamental electric field.

The results shown by the lines in Fig. 1(a) reveal for the plateau harmonics two main quantum paths labeled short (solid line) and long (dashed line). The experimental points clearly indicate that the short trajectory dominates the harmonic generation. The agreement between experimental and theoretical results justifies assigning the measured values to a constant intensity and shows that the temporal structure of the APT is dominated by the single-atom effects.

From the experimental results in Fig. 1(a), we can determine all the derivatives needed for the reconstruction of the APT intensity. They compare well with the SFA predictions, even though a better precision would be obtained with measurements for a larger number of intensities. In order to obtain the APT electric field, measurements of the chirp of the central harmonic are also needed, which were not performed in the present experiment. In addition, the fundamental field (including the chirp and CEP) must be determined. Below we present a reconstruction based on phase terms obtained from SFA calculations, including the experimentally yet unavailable term, $\Phi_{q_{0}}$, allowing us to discuss the absolute phase of the APT electric field. For the spectral amplitudes, we assume here a Gaussian distribution spanning from the 19th to the 37 th harmonics, with a full width at half maximum $\Delta q=$ 10. We also assume a Gaussian temporal envelope for both the infrared $(\tau=45 \mathrm{fs})$ and the harmonics, with equal durations of $\tau / \sqrt{3}$. We choose $\phi_{0}=0$.

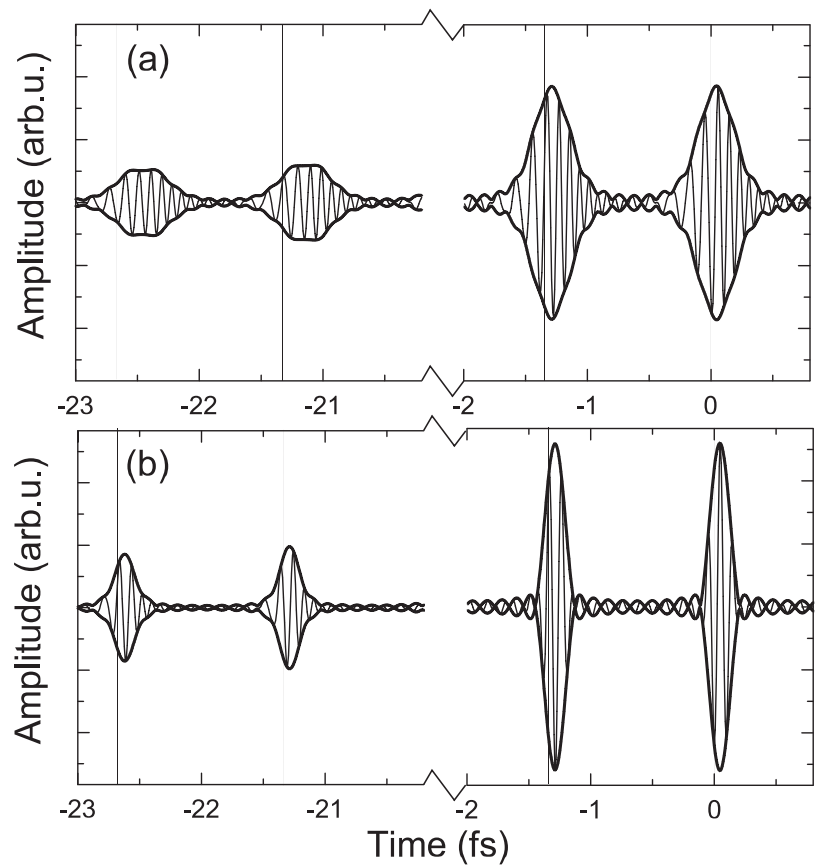

FIG. 2. Peak and half-peak sections of an APT in neon centered around the 27th harmonic of $800 \mathrm{~nm}$ with a $15 \mathrm{eV}$ bandwidth; (a) generated with a 45 fs-long Fourier transform-limited field; (b) generated with a positively chirped fundamental field (to eliminate the spacing change) and passing through a medium with negative group delay dispersion (to eliminate the attochirp).

Figure 2 shows a reconstruction for a peak intensity of $2 \times 10^{14} \mathrm{~W} / \mathrm{cm}^{2}$ in two cases: (a) when the pulse train is generated by a transform-limited laser pulse $\left(b_{0}=\delta=0\right)$ corresponding to the experimental results and (b) when the train is generated with an appropriately chosen positively chirped pulse, and passes through a medium of negative group delay dispersion $b_{0}=\gamma \partial^{2} \Phi / \partial q \partial I=1.5 \times$ $\left.10^{-3} \mathrm{fs}^{-2}, \delta=-1 / \omega^{2} \partial^{2} \Phi / \partial q^{2}=-1 \times 10^{-2} \mathrm{fs}^{2}\right)$. The figure illustrates how the timing and duration of the attosecond bursts vary along the train. As a consequence of the variation of the delay of the XUV bursts relative to the laser field, in the train generated with a transform-limited pulse the spacing between the attosecond bursts slightly increases with time [Fig. 2(a)]. To emphasize the effect, vertical lines guide the eye for even spacing. The position of the lines correspond to the maxima and minima of a transform-limited fundamental field. In the particular case examined here, where the central harmonic is "deep" inside the plateau region, the attosecond bursts appear just after the maxima or minima of the laser electric field, in agreement with the experimental result [Fig. 1(b)]. As was suggested earlier [7], the (small) spacing change can be corrected by using a fundamental field with a positive chirp $\left(b_{0}\right)$ [cf. Fig. 2(b)]. The attosecond pulses are chirped $[11,12]$, and the chirp increases symmetrically from the maximum of the pulse train, the pulses getting significantly longer on the wings. By propagation through a medium 


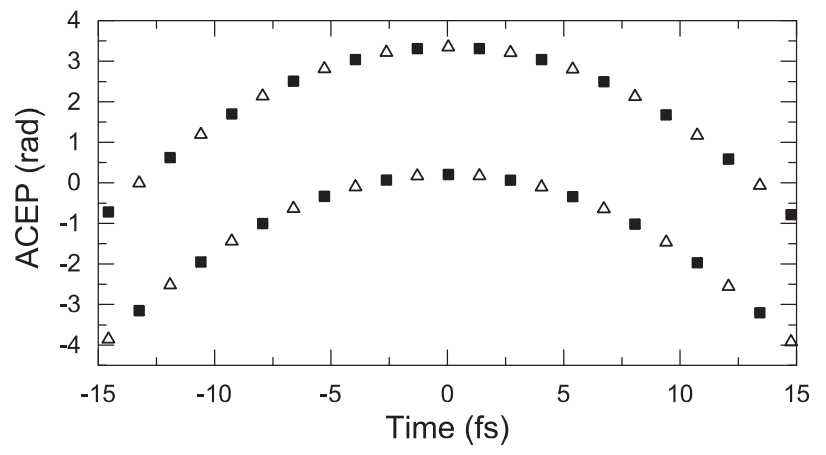

FIG. 3. ACEP of the attosecond bursts for trains generated at a peak intensity of $2 \times 10^{14} \mathrm{~W} / \mathrm{cm}^{2}$, plotted as a function of the position of the pulses in the train for a fundamental CEP; $\phi_{0}=0$ (squares) and $\phi_{0}=\pi$ (triangles).

with negative group delay dispersion, this attochirp can be reduced by the same amount for each pulse. In the example, we have chosen to compensate the chirp of the burst at the peak, leading to short, single cycle, 130 as pulses [Fig. 2(b)]. Even when the attosecond pulse at the peak is transform limited, as a result of the attochirp variation throughout the train, the pulses at the wing still show a small chirp.

For these pulses, close to the single cycle limit [17,22], the attosecond carrier envelope phase (denoted ACEP) becomes an important parameter. In Fig. 3 we plot the ACEP values of each of the bursts of the APT. The ACEP of the peak pulse is determined by the phase and group delay of the carrier component. For an infinite driving pulse, the ACEP of consecutive pulses in the train varies by a factor $\pi$, leading to two sets of points separated by $\pi$. For a finite IR pulse, there is a significant additional variation of the ACEP reflecting the variation of the phase and group delay with intensity. It does not, however, depend on the CEP or chirp of the fundamental. Mathematically, this is a consequence of the fact that the phase terms containing $\phi_{0}$ and $b_{0} t^{2} / 2$ are multiplied by $q$. In the conditions of the present work, i.e., for relatively long IR pulses, the fundamental CEP change does not modify the harmonic field, it simply shifts the attosecond bursts - together with the electric field - in time. Similarly, when a chirp is added to the fundamental, the position of the bursts changes, but the ACEP still follows the curves in Fig. 3. The CEP dependence of the ACEP found in [23] is a result of nonadiabatic effects, not discussed in the present work.

In conclusion, we have studied the behavior of the APT both on the attosecond and femtosecond time scales and have shown how the train can be experimentally characterized. A theoretical model is presented, using Taylor expansion both in time and frequency up to fourth order with coefficients bearing intuitive physical meaning. This allows us to discuss in simple terms the different temporal features, to reconstruct pulse-to-pulse variations of the APT, and to visualize the associated electric field. The coefficients, determined by RABITT measurements taken at different intensities, allow the reconstruction of the APT intensity. Together with a FROG/SPIDER determination of the central harmonic chirp and precise characterization of the fundamental field, it provides - apart from a phase constant - characterization of the attosecond pulse train electric field.

This research was supported by the Marie Curie European Program (MEIF-CT-2004-009268 and MRTNCT-2003-505138), the Integrated Infrastructure Initiative (RII3-CT-2003-506350, LASERLAB-EUROPE), the Knut and Alice Wallenberg Foundation, the Swedish Science Council, and a British EPSRC grant (GR/S22424/01).

*On leave from Department of Optics and Quantum Electronics, University of Szeged, Hungary.

${ }^{\dagger}$ Presently at Department of Physics and Astronomy, Louisiana State University, Baton Rouge, LA 708034001, USA.

[1] M. Hentschel et al., Nature (London) 414, 509 (2001).

[2] P. M. Paul et al., Science 292, 1689 (2001).

[3] Y. Kobayashi, T. Sekikawa, Y. Nabekawa, and S. Watanabe, Opt. Lett. 23, 64 (1998).

[4] T. Sekikawa, T. Ohno, T. Yamazaki, Y. Nabekawa, and S. Watanabe, Phys. Rev. Lett. 83, 2564 (1999).

[5] J. Norin et al., Phys. Rev. Lett. 88, 193901 (2002).

[6] T. Sekikawa, T. Katsura, S. Miura, and S. Watanabe, Phys. Rev. Lett. 88, 193902 (2002).

[7] J. Mauritsson et al., Phys. Rev. A 70, 021801(R) (2004).

[8] Y. Mairesse et al., Phys. Rev. Lett. 94, 173903 (2005).

[9] E. Cormier et al., Phys. Rev. Lett. 94, 033905 (2005).

[10] P. Agostini and L. F. DiMauro, Rep. Prog. Phys. 67, 813 (2004).

[11] Y. Mairesse et al., Science 302, 1540 (2003).

[12] S. Kazamias and Ph. Balcou, Phys. Rev. A 69, 063416 (2004).

[13] K. Varjú et al., J. Mod. Opt. 52, 379 (2005).

[14] F. Quéré, Y. Mairesse, and I. Itatani, J. Mod. Opt. 52, 339 (2005).

[15] Y. Mairesse and F. Quéré, Phys. Rev. A 71, 011401(R) (2005).

[16] J. Mauritsson et al., J. Phys. B 38, 2265 (2005).

[17] R. López-Martens et al., Phys. Rev. Lett. 94, 033001 (2005).

[18] Y. Mairesse et al., Phys. Rev. Lett. 93, 163901 (2004).

[19] P. Antoine, A. L'Huillier, and M. Lewenstein, Phys. Rev. Lett. 77, 1234 (1996).

[20] M. Bellini et al., Phys. Rev. Lett. 81, 297 (1998).

[21] M. Lewenstein, P. Salières, and A. L'Huillier, Phys. Rev. A 52, 4747 (1995).

[22] K. T. Kim, C. M. Kim, M.-G. Baik, G. Umesh, and C.H. Nam, Phys. Rev. A 69, 051805(R) (2004).

[23] G. Sansone et al., Phys. Rev. Lett. 94, 193903 (2005). 